\title{
New records of Hypoxylon hypomiltum and Hypomontagnella monticulosa from Pilikuththuwa lowland wet zone forest, Sri Lanka
}

\section{Palapathwala PLES, Daranagama DA*, Abeywickrama K and Kannangara BTSDP}

Department of Plant and Molecular Biology, Faculty of Science, University of Kelaniya, Kelaniya, Sri Lanka

Palapathwala PLES, Daranagama DA, Abeywickrama K, Kannangara BTSDP 2019 - Two new records of Hypoxylon hypomiltum and Hypomontagnella monticulosa from Pilikuththuwa lowland wet zone forest, Sri Lanka. Studies in Fungi 4(1), 142-152, Doi 10.5943/sif/4/1/17

\begin{abstract}
Species of Hypoxylon exhibit their highest diversity in the tropics. During an assessment of diversity of hypoxylaceous fungi in the lowland wet zone forest area in Sri Lanka, two new records were reported. Morphological identification of the collected samples was carried out using both macroscopic and microscopic characteristics. Genomic DNA was extracted using modified CTAB method and amplification of both ITS and $\beta$-tubulin genes were performed using ITS 1 and ITS 4 and T1 and T22 primers respectively. In the present study, recognition of species in Hypoxylon was carried out using a combined phylogenic analysis of ITS and $\beta$-tubulin molecular markers. Phylogenetic tree resulted from combined analysis comprised nine clades. According to the results, two new country records of Hypomontagnella monticulosa and Hypoxylon hypomiltum were recognized from Pilikuththuwa lowland wet zone forest.
\end{abstract}

Key words - Hypoxylaceae - Morphology - Phylogeny - Taxonomy

\section{Introduction}

Genus Hypoxylon (Bull.) was traditionally described in the family Xylariaceae (Ju \& Rogers 1996, Stadler 2011). The classical morphological concept of Ju \& Rogers (1996) was based on four main characters to distinguish Hypoxylon from other genera of Xylariaceae as (1) nodulisporium-like anamorphs, (2) unipartite stromata, (3) solid and homogeneous stromatal tissue below the perithecial layer and (4) stromata not upright. According to Ju \& Rogers (1996), the Hypoxylon genus was divided in to two sections, section Hypoxylon and Annulata. Later Hsieh et al. (2005) upgraded the Hypoxylon section Annulata into a genus level Annulohypoxylon, based on a multigene phylogeny and there on, Hypoxylon comprises only the species which were categorized under Hypoxylon section Hypoxylon.

With the current knowledge in taxonomy, phylogeny and chemical profiles, Hypoxylon was transferred to the family Hypoxylaceae (Ascomycota, Xylariales) with other related genera based on a multigene analysis and morphology of their asexual morphs (Daranagama et al. 2018, Wendt et al. 2018). Due to the simple stromatal anatomy, and the multi-DNA locus genealogy, the taxonomists identified that the species related to Hypoxylon monticulosum Mont. as an ancestral clade (Lambert et al. 2019). Lambert et al. (2019) accommodated a new genus Hypomontagnella following morphology and chemotaxonomic studies along with multi gene phylogeny. With this addition Hypomontagnella now comprised five species (Lambert et al. 2019). 
Target regions of the ribosomal DNA genes subjected to amplification and sequencing have become a promising tool in precise identification and characterization of fungi (Bitzer et al. 2008, Triebel et al. 2005). RNA coding genes such as the nuclear small and large subunit (nucSSU, nucLSU), the mitochondrial small subunit (mitSSU) and the internal transcribed spacer region (ITS) are routinely used in molecular phylogenetic studies of fungi (Lutzoni et al. 2004). However, due to the slow evolution of protein-coding genes such as RNA polymerases (e.g. RPB1, RPB2) and $\beta$-tubulin, they are more effective in inferring distant phylogenetic relationships (Hongsanan et al. 2017, Daranagama et al. 2018, Wendt et al. 2018).

Research at national level on taxonomy and phylogeny of Hypoxylon is scarce in Sri Lanka. However, few studies have carried out within Sri Lanka to characterize species of Hypoxylon (Kuhnert et al. 2014). Pilikuththuwa lowland wet zone forest, where the samples were collected, is a cave forest, which is located just 30 miles from Colombo, in Gampaha district. It covers approximately 200 acres and has approximately hundred small caves spread over. The vegetation of the forest includes the characters of lowland wet zone.

Present study made a major contribution towards the nomenclature of Sri Lankan hypoxylaceous fungal species while producing a stable classification for species based on polyphasic approach. Two new country records of Hypoxylon hypomiltum and Hypomontagnella monticulosa were found from Pilikuththuwa lowland wet zone forest and presented with illustrations, descriptions and phylogenetic analysis.

\section{Materials \& Methods}

\section{Specimen collection, examination and isolation}

Fresh specimens were collected from Pilikuththuwa lowland wet zone forest from decaying wood materials lying on the ground, during May-July 2018, based on external macro morphological features. Collected samples were placed in paper bags and they were brought to the laboratory for further observations. Both macroscopic and microscopic characters were observed in all collected specimens. Collected hypoxylaceous fungal species were morphologically characterized following the monograph published by Ju \& Rogers (1996). Surface colour, colony colour and $\mathrm{KOH}$ extractable pigments were recorded according to Rayner's colour chart (1970). Ascomata of collected Hypoxylon species were observed using the stereo microscope (Olympus SZ61 model, Philippines). Microscopic characters including asci and ascospores were observed using Phase Contrast Microscope (Olympus CX41 model, Tokyo, Japan). The ascus apical ring was stained using Melzer's reagent. Microphotography was carried out using Olympus DP 26 Mega Pixel camera fitted to the Phase Contrast Microscope (X400 magnification) (Olympus CX41 model, Tokyo, Japan). Measurements of stromata $(n=10)$, perithecia $(n=10)$, asci $(n=20)$ and ascospore $(n=20)$ were made from material mounted in water and the mean values were used in the description. Measurements were made with the Tarosoft (R) Image Frame Work Program and images used for figures were processed with Adobe Photoshop CC version 18.0 (Adobe Systems Inc.), The United States). In order to isolate fungi, the upper surface of any fruiting bodies were excised using sterilized scalpel blade under aseptic conditions. Pure cultures were obtained either from single spore or multispore isolation following the methods detailed by Chomnunti et al. (2014) and Daranagama et al. (2015). The cultures were maintained at $27{ }^{\circ} \mathrm{C}$ in MEA in the laboratory. Cultures and herbarium specimens of collected materials were deposited in Department of Botany, University of Kelaniya (UKBC and UKBH).

\section{DNA extraction, PCR and Sequencing}

DNA was extracted from cultures grown on malt extracted agar (MEA) plates. The total genomic DNA was extracted from 0.05 to $0.10 \mathrm{~g}$ of growing mycelium using modified CTAB protocol following Daranagama et al. (2015). Precipitated DNA was recovered by centrifugation at $12000 \mathrm{rpm}$ for $10 \mathrm{~min}$ followed by three steps of purification with $70 \%$ ethanol. The air dried, 
precipitate was dissolved in $25 \mu \mathrm{l}$ of nuclease free water and stored at $-20{ }^{\circ} \mathrm{C}$ until use for amplification reactions.

PCR amplifications were performed for ITS and $\beta$-tubulin genes using ITS 1 and ITS 4 (White et al. 1990) and T1 and T22 primer pairs (O’Donnell \& Cigelnik 1997) respectively. The total volume of $25 \mu \mathrm{L}$ reaction mixture [5 $\mu \mathrm{L}$ PCR buffer (1X), $1.5 \mu \mathrm{L} \mathrm{MgCl}_{2}(1.5 \mathrm{mM}), 0.5 \mu \mathrm{L}$ dNTP $(0.20 \mathrm{mM}), 01 \mu \mathrm{L}$ of each primer $(0.4 \mu \mathrm{M}), 0.25 \mu \mathrm{L}$ Taq polymerase $(1 \mathrm{U} / 1 \mu \mathrm{L})$ and $2.0 \mu \mathrm{L}$ template DNA (1:10 diluted)] was used for PCR with adjustments of components' volumes and concentration when needed. PCR was carried out in a thermal cycler (Veriti 96-Well Thermal Cycler, ABI Biosystems, USA). PCR conditions were summarized in Table 1. The amplified PCR products were visualized on 1\% agarose gel in $1 \mathrm{X}$ TBE containing ethidium bromide, using gel documentation system (QUANTUM ST5, Germany) against 100 bp and $1 \mathrm{~kb}$ DNA molecular weight markers (Promega, USA).

The PCR products were purified and DNA sequencing was performed at Genetech Pvt Ltd, Sri Lanka and Macrogen Inc. Seoul, Republic of Korea using the same primer pairs.

Table 1 PCR conditions used in PCR amplification.

\begin{tabular}{|c|c|c|}
\hline Gene & PCR primers (F/R) & PCR protocol \\
\hline ITS & ITS-1/ITS-4 & $\begin{array}{l}95{ }^{\circ} \mathrm{C}: 5 \mathrm{~min},\left(95{ }^{\circ} \mathrm{C}: 30 \mathrm{sec}, 54{ }^{\circ} \mathrm{C}: 30 \mathrm{sec}, 72{ }^{\circ} \mathrm{C}: 1.30 \mathrm{~min}, 37\right. \\
\text { cycles), } 72{ }^{\circ} \mathrm{C}: 10 \mathrm{~min}\end{array}$ \\
\hline$\beta$-tubulin & $\mathrm{T} 1 / \mathrm{T} 22$ & $\begin{array}{l}95{ }^{\circ} \mathrm{C}: 5 \mathrm{~min},\left(95{ }^{\circ} \mathrm{C}: 1 \mathrm{~min}, 54{ }^{\circ} \mathrm{C}: 1.50 \mathrm{~min}, 72{ }^{\circ} \mathrm{C}: 2 \mathrm{~min}, 35\right. \\
\text { cycles), } 72{ }^{\circ} \mathrm{C}: 10 \mathrm{~min}\end{array}$ \\
\hline
\end{tabular}

\section{Sequencing alignment and phylogenetic analysis}

Raw sequences were assembled using Bioedit 7.1.3.0. Sequence homologies for the assembled consensus sequences were analyzed using the BLASTn of the NCBI for the rough identification of fresh isolates used in the analysis. Representatives of Hypoxylon species downloaded from GenBank were used to infer phylogenetic relationships, including newly generated sequences from 2 strains using both ITS and $\beta$-tubulin. Sequences from 47 reliable strains were downloaded from GenBank (Table 2). Daldinia concentrica (CBS 113277) was used as the outgroup taxon.

Phylogenetic analysis was performed using a combined alignment of ITS and $\beta$ - tubulin gene sequences. The consensus sequences were then initially aligned using Clustal X v1.83 and optimized in Bioedit 7.1.3.0 where necessary.

The phylogenetic relationships were analyzed using Maximum likelihood (ML) analysis. ML gene-trees were estimated using RAxML-HPC2 on XSEDE tool (Stamatakis 2006, Stamatakis et al. 2008) available in the CIPRES Science Gateway platform (Miller et al. 2010). For the concatenated dataset all free modal parameters were estimated by RAxML with ML estimate of 25 per site rate categories. The RAxML software accommodated the GTRGAMMA model of nucleotide substitution with the additional options of modeling rate heterogeneity $(\Gamma)$ and proportion invariable sites (I). The resulting trees were viewed using Figtree v1.4.3. Bootstrap support (BS) values of ML analysis are indicated below or above the branches.

\section{Results}

\section{Phylogenetic analysis}

The combined phylogenetic dataset of ITS and $\beta$-tubulin contains 49 taxa with Daldinia concentrica as the out group taxon. The best scoring RAxML tree is shown in Fig. 3. According to the phylogram inferred from combining ITS and $\beta$-tubulin sequences (Fig. 3), Hypoxylon hypomiltum (UKBC026) from our collection clustered with H. hypomiltum (MUCL51845) with bootstrap support of $100 \%$ within subclade E. Further, subclade E comprised species of $H$. cercidicola, $H$. petriniae, $H$. carneum, $H$. perforatum, $H$. musceum, $H$. ochraceum, $H$. pilgerianum, 
H. samuelsii, and $H$. rubiginosum. Hypomontagnella monticulosa collected in the present study was clustered with the type species of Hypomontagnella monticulosa (MUCL54604) with 100\% bootstrap support within subclade G. Along with Hypomontagnella monticulosa, subclade G comprised species of Hypomontagnella submonticulosa and Hypomontagnella barbarensis as a separate clade. $H$. haematostroma, $H$. fragiforme, $H$. howeanum, $H$. ticinense, $H$. subgilvum and $H$. rickii clustered within clade $\mathrm{C}$ with high bootstrap supports. Clade D comprised of species of $\mathrm{H}$. erythrostroma, $\mathrm{H}$. lenormandii, $H$. fendleri, $H$. rutilum and $H$. crocopeplum. $H$. trugodes, $H$. fuscopurpureum, $H$. aeruginosum, $H$. begae, $H$. vinosopulvinatum, and $H$. griseobrunneum clustered within clade $H$ where $H$. pulicicidum, $H$. investiens and $H$. lateripigmentum clustered in clade I with high bootstrap support.

\section{Taxonomy}

Hypomontagnella monticulosa (Mont.) Sir, L. Wendt \& C. Lambert, Mycological Progress 18 (12): 190 (2018)

Index Fungorum number: IF 165810; Faces of fungi number: FoF 06136

Fig. 1

Saprobic on decaying dicot branches. Stromata superficial, effused-pulvinate, forming compact mass, surface brown to black, conspicuous, $\mathrm{KOH}$ extractable pigments Vinaceous purple (101). Perithecia $211.23( \pm 14.99) \times 184.10( \pm 7.74) \mu \mathrm{m}$, spherical to hemispherical, Asci $46.56( \pm 3.99)$ $\mu \mathrm{m}$ in total length $\times 1.78( \pm 0.07) \mu \mathrm{m}$ broad, cylindrical, 8-spored, spore bearing part $18.03( \pm$ 2.01) $\mu \mathrm{m}$, stipe $28.54( \pm 2.28) \mu \mathrm{m}$, with $\mathrm{J}+$, wedged-shaped apical ring bluing in Melzer's reagent. Ascospores $3.88( \pm 0.11) \times 1.84( \pm 0.06) \mu \mathrm{m}$, light brown to brown, ellipsoidal-inequilateral, broadly rounded ends, uniseriate, unicellular, sigmoid germ slit, nearlt spore - length.

Culture characteristics - Culture on MEA covering $9 \mathrm{~cm}$ plate within $45-50$ days at $27{ }^{\circ} \mathrm{C}$, Pale vinaceous (85) to Vinaceous buff (86) mycelia, with dense Pale vinaceous (85) center, reverse Umber (19), Dark brick (60) center with Hasel (88).

Known distribution - Brazil, Colombia, Guyana, French Guiana, Mauritius, Mexico, Paraguay, Philippines, Sri Lanka, Taiwan, USA

Material examined - Sri Lanka, Western Province, Gampaha district, Pilikuththuwa lowland wet zone forest, on decaying dicot wood, on the track to caves, $7^{\circ} 03^{\prime} 50.2119^{\prime \prime} \mathrm{N}$ and $8^{\circ} 03^{\text {' }}$ 01.1014” E, 29 June 2018, herbarium = UKBH017, Daranagama and Palapathwala, HYXL 017, living culture $=$ UKBC017.

Notes - Hypomontagnella monticulosa which was previously accommodated in Hypoxylon was recently upgraded into a separate genus (Lambert et al. 2019). Our collection identified as $H$. monticulosa has morphological similarities with the described isotype of $\mathrm{H}$. monticulosa in

Lambert et al. (2019) including vinaceous young stromata and $\mathrm{KOH}$-extractable pigments, papillate ostioles, asci with discoid apical ring and ascospores with more or less spore-length sigmoid germ slit. However, the size of ascospores from our collection is comparatively smaller than those previously described where our collection had $4 \mu \mathrm{m}$ and previous studies had $5-7 \mu \mathrm{m}$. Three collections of $H$. monticulosa from the same locality were compared further, where the ascospores of all three materials fall within the same range $(3-5 \mu \mathrm{m})$ of the described material UKBH017. Hypomontagnella monticulosa identified from Pilikuththuwa forest clustered with its reference strain (H. monticulosa, MUCL54604) from French Guiana with 100\% bootstrap support. Thus we conclude that the difference of the ascospores measurements could be due to environmental and geographical factors.

Hypoxylon hypomiltum Mont., Annls Sci. Nat., Bot., sér. 2 13: 356 (1840).

Fig. 2

Index Fungorum number: IF 158066; Facesoffungi number: FoF 06137

Saprobic on decaying dicot twigs. Stromata superficial, sessile, effused-pulvinate, conspicuous, hemispherical, dense, forming compact mass, discoid, surface black, $\mathrm{KOH}$ extractable pigments Citrine (13), Oehrecous (44). Perithecia $305.61( \pm 27.22) \times 250.21( \pm 5.56) \mu \mathrm{m}$, hemispherical to spherical. Asci spore bearing part $47.18( \pm 0.68) \mu \mathrm{m}$, cylindrical, 8-spored, 81.75 $( \pm 0.49) \mu \mathrm{m}$ in total length $\times 5.93( \pm 0.17) \mu \mathrm{m}$ broad, stipe $34.57( \pm 0.78) \mu \mathrm{m}$ with $\mathrm{J}+$, discoid. 
Ascospores $7.99( \pm 0.10) \times 3.26( \pm 0.20) \mu \mathrm{m}$, dark brown, ellipsoidal-equilateral, uniseriate, unicellular, broadly rounded ends, straight germ slit, spore-length.

Table 2 List of taxa used in the phylogenetic study with their strain numbers, origin, GenBank accession numbers and references.

\begin{tabular}{|c|c|c|c|c|c|}
\hline \multirow{2}{*}{ Species } & \multirow{2}{*}{ Strain Number } & \multirow{2}{*}{ Origin } & \multicolumn{2}{|c|}{ GenBank accession numbers } & \multirow{2}{*}{ Reference } \\
\hline & & & ITS & $\beta$-tubulin & \\
\hline Hypoxylon aeruginosum & MUCL 54620 & French Guiana & KC968941 & KC977305 & Kuhnert et al. 2014 \\
\hline Hypoxylon begae & BCRC 34051 & Hawaii (USA) & JN660820 & AY951704 & Kuhnert et al. 2014 \\
\hline Hypoxylon carneum & MUCL 54177 & France & KY610400 & KX271270 & Wendt et al. 2018 \\
\hline Hypoxylon cercidicola & CBS 119009 & France & CBS 119009 & KC977263 & Wendt et al. 2018 \\
\hline Hypoxylon crocopeplum & CBS 119004 & France & KC968907 & KC977268 & Wendt et al. 2018 \\
\hline Hypoxylon erythrostroma & MUCL 53759 & Maritinique & KC968910 & KC977296 & Kuhnert et al. 2014 \\
\hline Hypoxylon fendleri & MUCL 54792 & French Guiana & KF234421 & KF300547 & Wendt et al. 2018 \\
\hline Hypoxylon fragiforme & MUCL 51264 & Germany & KC477229 & KX271282 & Wendt et al. 2018 \\
\hline Hypoxylon fuscopurpureum & BCRC 34067 & USA & JN979421 & AY951721 & Kuhnert et al. 2014 \\
\hline Hypoxylon fuscum & CBS 113049 & France & KY610401 & KX271271 & Wendt et al. 2018 \\
\hline Hypoxylon griseobrunneum & CBS 331.73 & India & KY610402 & KC977303 & Wendt et al. 2018 \\
\hline Hypoxylon haematostroma & MUCL 53301 & Martinique & KC968911 & KC977291 & Wendt et al. 2018 \\
\hline Hypoxylon howeanum & MUCL 47599 & Germany & AM749928 & KC977277 & Wendt et al. 2018 \\
\hline Hypoxylon hypomiltum & MUCL 51845 & Guadeloupe & KY610403 & KX271249 & Wendt et al. 2018 \\
\hline Hypoxylon hypomiltum & UKBC 026 & Sri Lanka & MK898827 & MK905762 & This study \\
\hline Hypoxylon investiens & CBS 118183 & Malaysia & KC968925 & KC977270 & Wendt et al. 2018 \\
\hline Hypoxylon lateripigmentum & MUCL 53304 & Martinique & KC968933 & KC977290 & Wendt et al. 2018 \\
\hline Hypoxylon lenormandii & CBS 119003 & Ecuador & KC968943 & KC977273 & Wendt et al. 2018 \\
\hline Hypomontagnella barbarensis & STMA 14081 & Argentina & MK131720 & MK135893 & Lambert et al. 2019 \\
\hline Hypomontagnella monticulosa & MUCL 54604 & French Guiana & KY610404 & KX271273 & Wendt et al. 2018 \\
\hline Hypomontagnella monticulosa & UKBC 017 & Sri Lanka & MK898826 & - & This study \\
\hline Hypomontagnella submonticulosa & CBS 115280 & France & KC968923 & KC977267 & Kuhnert et al. 2014 \\
\hline Hypoxylon musceum & MUCL 53765 & Guadeloupe & KC968926 & KC977280 & Wendt et al. 2018 \\
\hline Hypoxylon nicaraguense & CBS 117739 & Burkina Faso & AM749922 & KC977272 & Kuhnert et al. 2014 \\
\hline Hypoxylon ochraceum & MUCL 54625 & Martinique & KC968937 & KC977300 & Wendt et al. 2018 \\
\hline Hypoxylon papillatum & ATCC 58729 & USA & KC968919 & KC977258 & Wendt et al. 2018 \\
\hline Hypoxylon perforatum & CBS 115281 & France & KY610391 & KX271250 & Wendt et al. 2018 \\
\hline Hypoxylon petriniae & CBS 114746 & France & KY610405 & KX271274 & Wendt et al. 2018 \\
\hline Hypoxylon pilgerianum & STMA 13455 & Martinique & KY610412 & KY624315 & Wendt et al. 2018 \\
\hline
\end{tabular}


Table 2 Continued.

\begin{tabular}{|c|c|c|c|c|c|}
\hline \multirow{2}{*}{ Species } & \multirow{2}{*}{ Strain number } & \multirow{2}{*}{ Origin } & \multicolumn{2}{|c|}{ GenBank accession numbers } & \multirow{2}{*}{ Reference } \\
\hline & & & ITS & $\beta$-tubulin & \\
\hline Hypoxylon porphyreum & CBS 119022 & France & KC968921 & KC977264 & Wendt et al. 2018 \\
\hline Hypoxylon pulicicidum & CBS 122622 & Martinique & JX183075 & JX183072 & Wendt et al. 2018 \\
\hline Hypoxylon rickii & MUCL 53309 & Martinique & KC968932 & KC977288 & Wendt et al. 2018 \\
\hline Hypoxylon rubiginosum & MUCL 52887 & Germany & KC477232 & KY624311 & Wendt et al. 2018 \\
\hline Hypoxylon rutilum & BCRC 34092 & France & JF908084 & AY951752 & Kuhnert et al. 2014 \\
\hline Hypoxylon samuelsii & MUCL 51843 & Guadeloupe & KC968916 & KC977286 & Wendt et al. 2018 \\
\hline Hypoxylon subgilvum & BCRC 34094 & Hawai (USA) & JQ009314 & AY951754 & Kuhnert et al. 2014 \\
\hline Hypoxylon ticinense & CBS 115271 & France & JQ009317 & AY951757 & Hsieh et al. 2005 \\
\hline Hypoxylon trugodes & MUCL 54794 & Sri Lanka & KF234422 & KF300548 & Wendt et al. 2018 \\
\hline Hypoxylon ulmophilum & BCRC 34100 & Russia & JQ009320 & AY951760 & Kuhnert et al. 2014 \\
\hline Hypoxylon vinosopulvinatum & BCRC 34101 & Taiwan & JQ009321 & AY951761 & Kuhnert et al. 2014 \\
\hline Hypoxylon vogesiacum & CBS 115273 & France & KC968920 & KX271275 & Wendt et al. 2018 \\
\hline Annulohypoxylon annulatum & CBS 140775 & Texas & KY610418 & KX376353 & Wendt et al. 2018 \\
\hline Annulohypoxylon atroroseum & ATCC 76081 & Thailand & AJ390397 & DQ840083 & Wendt et al. 2018 \\
\hline Annulohypoxylon michelianum & CBS 119993 & Spain & KX376320 & KX271239 & Wendt et al. 2018 \\
\hline Annulohypoxylon moriforme & CBS123579 & Martinique & KX376321 & KX271261 & Wendt et al. 2018 \\
\hline Annulohypoxylon nitens & MFLUCC 12.0823 & Thailand & KJ934991 & KJ934993 & Wendt et al. 2018 \\
\hline Annulohypoxylon stygium & MUCL 54601 & French Guiana & KY610409 & KX271263 & Wendt et al. 2018 \\
\hline Annulohypoxylon truncatum & CBS 140778 & Texas & KY610419 & KX376352 & Wendt et al. 2018 \\
\hline Daldinia concentrica & CBS 113277 & Germany & AY616683 & KC977274 & $\begin{array}{l}\text { Triebel et al. 2005, } \\
\text { Kuhnert et al. } 2014\end{array}$ \\
\hline
\end{tabular}

Culture characteristics - Culture on MEA covering $9 \mathrm{~cm}$ plate within 20-22 days at $27{ }^{\circ} \mathrm{C}$, smooth, soft, Velvety, Pale vinaceous (85), reverse Dark vinaceous (82), Sepia (63), with symmetric edges.

Known distribution - Brazil, Dominican Republic, French Guiana, Mexico, Puerto Rico, Taiwan, USA

Material examined - Sri Lanka, Western Province, Gampaha district, Pilikuththuwa forest, on the track to caves, $7^{\circ} 03^{\prime} 50.2135^{\prime}$ ' N and $8^{\circ} 03^{\prime}$ 01.1037” E, 20 July 2018, herbarium = UKBH026, Daranagama and Palapathwala, HYXL 026, living culture = UKBC026.

Notes - Hypoxylon hypomiltum from our culture collection has similar characters with previously studied species. Both of them have effusedpulvinate stromata, similar lengths in perithecia, more or less similar lengths in asci. Also both of them have equilateral, spore length germ slit in ascospores. The species identified as Hypoxylon hypomiltum from Pilikuththuwa forest clustered with its reference strain (H. hypomiltum, MUCL52887) from Guadeloupe with 100\% bootstrap support. 




Fig. 1 - Morphological features of Hypomontagnella monticulosa (HYXL 017). a Appearance of stromata on substrate (shown by arrow head). b KOH extractable pigments. c Cross section of stromata showing ascomata (shown by arrow heads). d Ascospore (germ slit shown by arrow head). e, f, g Asci (germ slit shown by arrow head). h Asci in Melzer's reagent (J+ apical ring shown by arrow head). i Upper surface of the culture. $\mathrm{j}$ Lower surface of the culture (Culture on MEA after 45 days). 


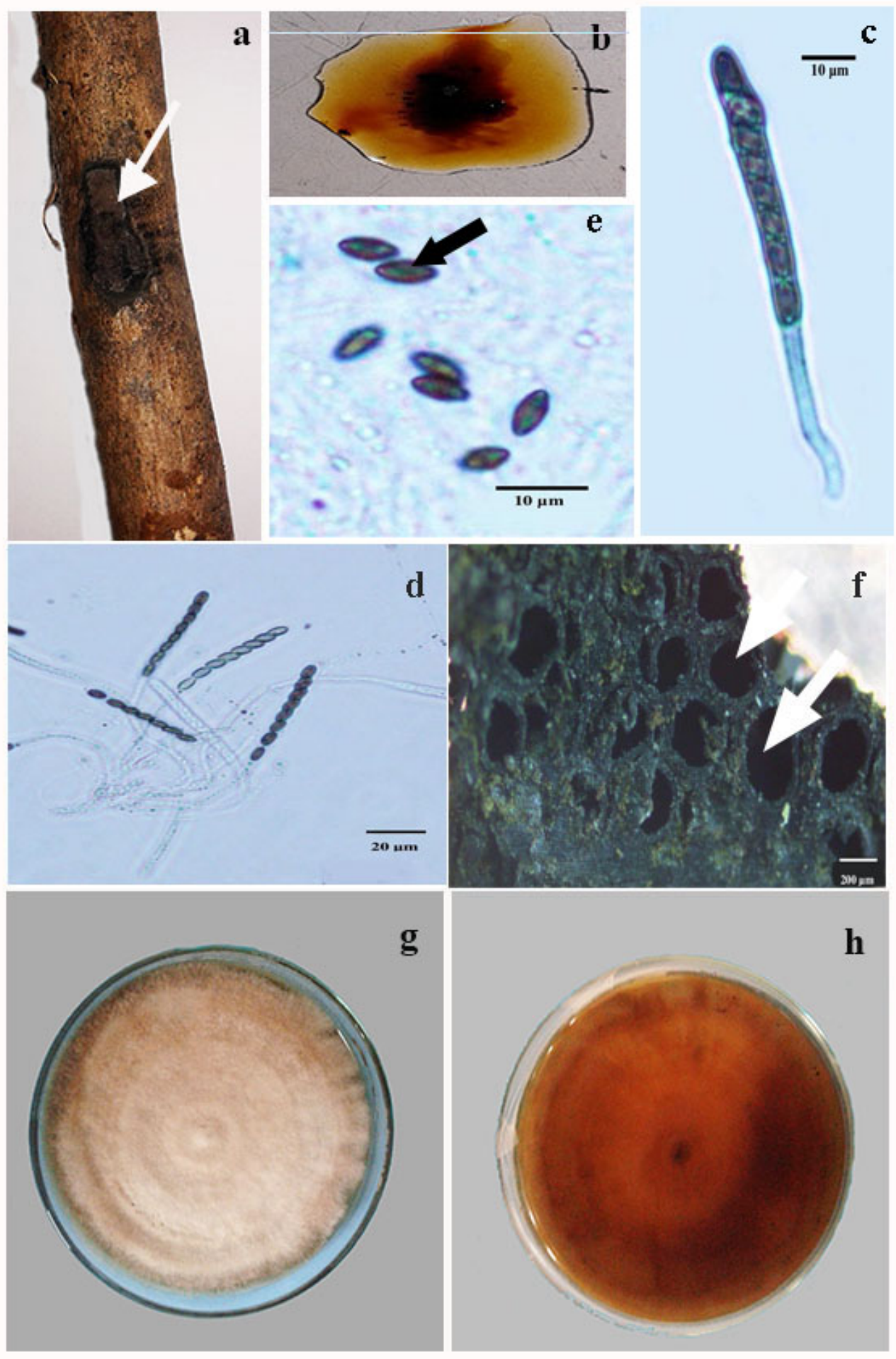

Fig. 2 - Morphological features of Hypoxylon hypomiltum (HYXL 026). a Appearance of stromata on substrate (shown by arrow head). b KOH extractable pigments. c, d Asci. e Ascospore (germ slit shown by arrow head). f Cross section of stromata showing ascomata (shown by arrow heads). g Upper surface of the culture. h Lower surface of the culture (Culture on MEA after 20 days).

\section{Discussion}

For the preliminary identification and characterization of the collected samples, morphological characters of sexual morph were considered in this present study. Depending on the above features, the identification of the species was carried out using keys provided by Ju \& Rogers (1996). As a result, two species, Hypomontagnella monticulosa and Hypoxylon hypomiltum were recorded from Pilikuththuwa lowland wet zone forest. 
Application of RNA coding genes and ITS was used for molecular phylogeny of fungi (Lutzoni et al. 2004). But in very recent past, number of studies (Daranagama et al. 2018, Wendt et al. 2018) used protein coding genes such as $\alpha$-actin and $\beta$-tubulin, because the usage of protein coding genes proved to be more efficient in phylogenetic studies.

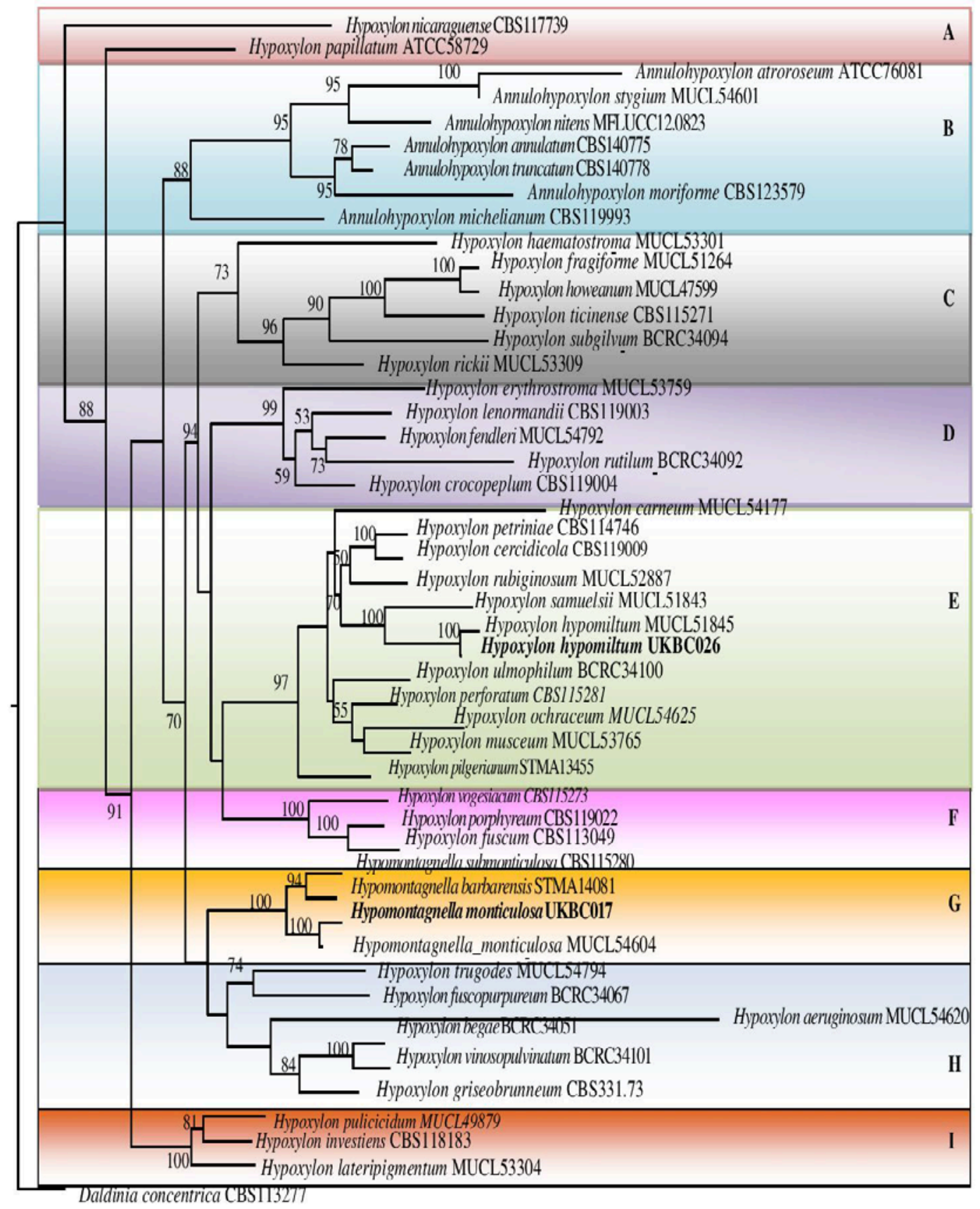

Fig. 3 - Phylogram constructed with RAxML using combined dataset of ITS and $\beta$-tubulin sequences. Bootstrap support values $\geq 50 \%$ are indicated below or above the nodes. Daldinia concentrica was used as the out group taxon. Sequences obtained from current study are shown in bold.

Single gene tree of ITS did not provide a good resolution of terminal taxa. Even though, single gene tree of $\beta$-tubulin had a low resolution within the species, it was more informative than single gene tree of ITS. Due to the poor resolution of both single gene trees, the combined gene tree 
of ITS and $\beta$-tubulin was used. As expected, the combined analysis was more successful and it provided better resolution within species. Thus, the combined analysis was more informative and successfully used in phylogenetic studies to infer the phylogenetic relationship among species.

The combined phylogenetic tree comprised nine clades including Annulohypoxylon as a separate clade - clade B with all Annulohypoxylon strains.

Sri Lanka, though a small country, has a great deal of biodiversity of fungi. However, information available on Sri Lankan fungi is scattered. The studies on fungal diversity were initiated before 1800s in Sri Lanka, and the first Sri Lankan fungi to be recorded were Peziza ceylonische and $P$. lembosa (Karunarathna et al. 2012). From there, number of mycologists carried out several studies on Sri Lankan fungi (Karunarathna et al. 2012). According to Karunarathna et al. (2012), current statistics suggest that there could be as many as 25,000 species, of which only a little more than 2,000 are presently known.

\section{References}

Bitzer J, Læssøe T, Fournier J, Kummer V et al. 2008 - Affinities of Phylacia and the daldinoid Xylariaceae, inferred from chemotypes of cultures and ribosomal DNA sequences. Mycological Research 112, 251-270.

Chomnunti P, Hongsanan S, Aguirre-Hudson B, Tian Q et al. 2014 - The sooty moulds. Fungal Diversity 66, 1-36.

Daranagama DA, Camporesi E, Tian Q, Liu X et al. 2015 - Anthostomella is polyphyletic comprising several genera in Xylariaceae. Fungal Diversity 73, 203-238.

Daranagama DA, Hyde KD, Sir EB, Thambugala KM et al. 2018 - Towards a natural classification and backbone tree for Graphostromataceae, Hypoxylaceae, Lopadostomataceae and Xylariaceae. Fungal Diversity 88, 1-165.

Hongsanan S, Maharachchikumbura SSN, Hyde KD, Samarakoon M et al. 2017 - An updated phylogeny of Sordariomycetes based on phylogenetic and molecular clock evidence. Fungal Diversity 84, 25-41.

Hsieh HM, Ju YM, Rogers JD. 2005 - Molecular phylogeny of Hypoxylon and closely related genera. Mycologia 97, 844-865.

Ju YM, Rogers JD. 1996 - A revision of the genus Hypoxylon. Mycologia Memoirum No. 20. APS Press, St. Paul.

Karunarathna SC. 2012 - Current status of knowledge of Sri Lankan mycota. Current Research in Environmental \& Applied Mycology 2, 18-29.

Kuhnert E, Heitkämper S, Fournier J, Surup F, Stadler M. 2014 - Hypoxyvermelhotins A-C, new pigments from Hypoxylon lechatii sp. nov. Fungal Biology 118, 242-252.

Lambert C, Wendt L, Hladki AI, Stadler M, Sir EB. 2019 - Hypomontagnella (Hypoxylaceae): a new genus segregated from Hypoxylon by a polyphasic taxonomic approach. Mycological Progress 18, 187-201.

Lutzoni F, Kauff F, Cox CJ, McLaughlin D et al. 2004 - Assembling the fungal tree of life: progress, classification, and evolution of subcellular traits. American Journal of Botany 91, 1446-1480.

Miller MA, Pfeiffer W, Schwartz T. 2010 - Creating the CIPRES Science Gateway for inference of large phylogenetic trees. In Gateway Computing Environments Workshop (GCE). 1-8

O’Donnell K, Cigelnik E. 1997 - Two divergent intragenomic rDNA ITS2 types within a monophyletic lineage of the fungus Fusarium are nonorthologous. Molecular Phylogenetics and Evolution 7, 103-116.

Rayner RW. 1970 - A mycological colour chart. Commonwealth Mycological Institute, Kew and British Mycological Society.

Stadler M. 2011 - Importance of secondary metabolites in the Xylariaceae as parameters for assessment of their taxonomy, phylogeny, and functional biodiversity. Current Research in Environmental \& Applied Mycology 1, 75-133. 
Stamatakis A. 2006 - RAxML-VI-HPC: maximum likelihood-based phylogenetic analyses with thousands of taxa and mixed models. Bioinformatics 22, 2688-2690.

Stamatakis A, Hoover P, Rougemont J. 2008 - A rapid bootstrap algorithm for the RAxML web servers. Systematic Biology 57, 758-771.

Triebel D, Peršoh D, Wollweber H, Stadler M. 2005 - Phylogenetic relationships among Daldinia, Entonaema and Hypoxylon as inferred from ITS nrDNA sequences. Nova Hedwigia 80, 2543.

Wendt L, Sir EB, Kuhnert E, Heitkämper S et al. 2018 - Resurrection and emendation of the Hypoxylaceae, recognised from a multigene phylogeny of the Xylariales. Mycological Progress 17, 115-154.

White TJ, Bruns TD, Lee S, Taylor JW. 1990 - Amplification and direct sequencing of fungal ribosomal RNA for phylogenetics. In: Innis MA, Gelfand, D. H., Sninsky, J. J., White, T. J., eds. PCR protocols: a guide to methods and applications. San Diego, California Academic Press. 315-322. 